\title{
Sulfur Speciation in an Arable Soil as Affected by Sample Pretreatments and Sewage Sludge Application
}

\author{
Lei Luo \\ Chuang Xu \\ State Key Lab. of Environ. Chemistry \\ and Ecotoxicology \\ Research Center for Eco-Environ. Sciences \\ Chinese Academy of Sciences \\ Beijing 100085 \\ China

\section{Yibing Ma} \\ National Soil Fertility and Fertilizer Effects \\ Long-term Monitoring Network \\ Inst. of Agricultural Resources and \\ Regional Planning \\ Chinese Academy of Agricultural Sciences \\ Beijing 100081 \\ China
}

\section{Lei Zheng \\ Lijuan Liu \\ State Key Lab. of Synchrotron Radiation \\ Inst. of High Energy Physics \\ Chinese Academy of Sciences \\ Beijing 100049 \\ China}

\section{Jitao Lv}

Shuzhen Zhang*

State Key Lab. of Environ. Chemistry and Ecotoxicology

Research Center for Eco-Environ. Sciences Chinese Academy of Sciences Beijing 100085

China
Sulfur speciation under the influence of sample pretreatment and sewage sludge (SS) application in a continuously SS-amended soil was assessed by wetchemical fractionation and $S K$-edge XANES spectroscopy. Distinct differences in $\mathrm{S}$ speciation among the following pretreatments including fresh, air-drying, anoxic freeze-drying, oven-drying, and conditioning (incubation at room temperature and $75 \%$ of water holding capacity for $2 \mathrm{wk}$ ) were detected in both topsoils and subsoils. Air-drying significantly increased available $S$ content but decreased C-bonded S content in the soils compared with anoxic freeze-drying. Oven-drying and conditioning pretreatments showed a similar effect on $\mathrm{S}$ fractions to air-drying in the soils but to a lesser extent. X-ray adsorption nearedge structure analysis showed that both reduced and intermediate organic $S$ tended to decrease while oxidized $S$ obviously increased in the air-dried samples. In contrast, the conditioning pretreatment facilitated the transformation of intermediate $\mathrm{S}$ into reduced organic S. Continuous application of SS significantly increased total soil S, C-bonded S, and residual S contents but decreased available $S$ content in the topsoil. Meanwhile, XANES analysis indicated that reduced organic $S$ was markedly increased (over $50 \%$ of total $S$ ) in the SS-amended topsoil. No obvious effect of the application of SS on S speciation in the subsoil was observed except for the facilitated transportation of $S$ into lower depth. Overall, our results suggest that anoxic atmospheres during the entire pretreatment process from sampling to drying, grinding, and analysis is crucial for the accurate speciation analysis of $S$ in soils, and the anoxic freezedrying is, therefore, highly recommended.

\section{Abbreviations: DOC, dissolved organic C; SS, sewage sludge; TOC, total organic C.}

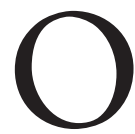
ver the last decade, $S$ deficiency has been recognized as a constraint to crop production all over the world due to the reduction of atmospheric $\mathrm{SO}_{2}$ emissions, the application of non-S-containing fertilizers, and the increase of crop yields (Kirchmann et al., 1996; Zhao et al., 2006). Previous studies have shown that application of organic manure cannot only significantly increase soil $S$ content but also markedly affect the distribution of $S$ species in soils (Yang et al., 2007; Förster et al., 2012). However, little information is available on the effect of SS on S speciation in soils although SS contains a large pool of potentially mineralizable S (Kirchmann et al., 1996) and is often applied to agricultural soils as soil amendments (Li et al., 2012). To obtain a better understanding of soil S supply capacity to crops and to assess the impact of SS application on $S$ speciation dynamics require detailed information on the speciation and transformation of $S$ in soils.

Sulfur in soils exists in both organic and inorganic forms with different oxidation states, ranging from -2 to +6 . The speciation of $S$ is relevant for many biogeochemical processes, including the cycling of nutrient (Solomon et al., 2005)

This article has supplemental material available.

Soil Sci. Soc. Am. J. 78:1615-1623

doi:10.2136/sssaj2013.11.0506

Received 27 Nov. 2013

*Corresponding author (szzhang@rcees.ac.cn).

(C) Soil Science Society of America, 5585 Guilford Rd., Madison WI 53711 USA

All rights reserved. No part of this periodical may be reproduced or transmitted in any form or by

any means, electronic or mechanical, including photocopying, recording, or any information storage and retrieval system, without permission in writing from the publisher. Permission for printing and for reprinting the material contained herein has been obtained by the publisher. 
and toxic elements (e.g., Hashimoto and Yamaguchi, 2013). Meanwhile, it could be markedly affected by environmental redox potential and pretreatment condition (Prietzel et al., 2009). It is suggested that reduced organic $S$ compounds are more stable than reduced inorganic $S$ compounds in soils (Novák and Wieder, 1992; Hutchison et al., 2001), whereas our recent results indicated that $\mathrm{C}$-bonded $\mathrm{S}$ could be substantially degraded in air-dried soil samples after storage in capped bottles at ambient atmosphere over a period of $20 \mathrm{yr}$ (Xu et al., 2014). Prietzel et al. (2009) investigated the effects of anoxic and oxic sample pretreatments on $S$ speciation in undisturbed forest soils and observed distinct differences in the distribution of $S$ species between anoxic freeze-dried and oxic oven-dried samples. Although some studies have acknowledged the influence of sample drying on $S$ speciation in soils (Wieder et al., 1996; Prietzel et al., 2007), a number of researchers just adopted airdried (Solomon et al., 2005; Zhao et al., 2006; Solomon et al., 2011) or even over-dried (Hinckley et al., 2011) samples for $S$ speciation analysis. To simulate real states of field soils, another pretreatment by remoistening air-dried soils and incubating for over $2 \mathrm{wk}$ at room temperature (referred to "conditioning" hereafter) was adapted to minimize the effects of soil handling and preparation and to stabilize soil microbial activities (Ghani et al., 1992; Tan et al., 1994; Pamidi et al., 2001). To date, detailed molecular-level information on the influence of different pretreatments on $S$ speciation in aerated arable soils is still lacking. A standard procedure of sample pretreatment for $S$ speciation analysis should, therefore, be developed with the purpose of accurate quantification of the oxidation states and speciation of $S$ in soils.

Determination of $S$ speciation in soils usually involves traditional wet-chemical fractionation and noninvasive synchrotron-based S K-edge XANES spectroscopy. Particularly the XANES spectroscopy, as an in situ technique, can circumvent the limitations such as alterations of oxidation states that degradative wet-chemical extraction techniques usually suffered from, and directly identify and quantify the oxidation states and moieties of $S$ in a variety of environmental and geochemical samples, even under field-moist conditions (Xia et al., 1998; Solomon et al., 2005; Prietzel et al., 2009; Solomon et al., 2011). However, the chemical fractionation is still useful to reflect the availability information of $S$ and to qualify the contents of inorganic and organic $S$ in soils and is, thus, currently irreplaceable (Yang et al., 2007). It is expected that XANES analysis coupled with chemical fractionation can well discern the changes of $S$ speciation in soils under the influence of various pretreatments and facilitate the construction of standard methods for soil pretreatments.

The objectives of the present study were to evaluate the effects of different pretreatments, including fresh field-moist, anoxic freeze-dried, oxic air-dried, oven-dried, and conditioned samples, on the speciation of $S$ in an agriculture soil and to explore the distribution and transformation of $S$ speciation in the soil under the influence of application of SS. For this purpose, a fluvo-aquic soil amended with SS since 2006 was selected and a combination of synchrotron based XANES spectroscopy and wet-chemical fractionation analyses was applied. The results are expected to be helpful to standardize the pretreatment of soils relating to redox-sensitive elements and to understand the speciation evolution of $S$ in SS-amended soils.

\section{MATERIALS AND METHODS Site and Soil}

A fluvo-aquic soil (Inceptisol) used in the study was collected from the Dezhou Experimental Station $\left(37^{\circ} 20^{\prime} \mathrm{N}\right.$ lat; $116^{\circ} 38^{\prime}$ E long), Chinese Academy of Agricultural Sciences. The soil has been continuously applied with SS (from the Beijing Sludge Disposal Plant) at a rate of $36.0 \mathrm{t} \mathrm{ha}^{-1}$ (dry wt.) per year in block experiments since 2006. Basal fertilizers including $360 \mathrm{~kg} \mathrm{~N} \mathrm{ha}^{-1}$ (in urea), $39.3 \mathrm{~kg} \mathrm{P} \mathrm{ha}^{-1}$ (in Ca superphosphate), and $99.6 \mathrm{~kg} \mathrm{~K} \mathrm{ha}^{-1}$ (in $\mathrm{K}_{2} \mathrm{SO}_{4}$ ) were applied per year. By comparison, a control soil, fertilized only with the same amounts of $\mathrm{P}$ and $\mathrm{K}$ fertilizers but without $\mathrm{SS}$ or $\mathrm{N}$ fertilizer, was also collected in the block experiments. The crop rotation was winter wheat (Triticum aestivum L.)-summer maize (Zea mays L.) each year at the station. Both surface $(0-20 \mathrm{~cm})$ and subsurface $(20-40$ $\mathrm{cm}$ ) soils were collected after the harvest of wheat (June 2013) to examine the distribution and transformation of $S$ species along the soil profiles.

\section{Sampling and Sample Pretreatments}

To minimize artificial sample changes by oxidation or microbial transformation, strictly anoxic conditions throughout the entire process from sampling to pretreatment were maintained as suggested by Prietzel et al. (2009). Briefly, the soil samples were taken with a steel auger and quickly loaded into sealed plastic bags and kept in a foam incubator filled with solid $\mathrm{CO}_{2}$. Within $6 \mathrm{~h}$ after sampling, an aliquot of fresh field-moist soil samples from the incubator was directly stored in a freezer under $-25^{\circ} \mathrm{C}$. An aliquot was freeze-dried under a vacuum condition, finely ground in a glove box under $\mathrm{N}_{2}$ atmosphere $(110 \mathrm{kPa})$, and stored as were the fresh samples. Another two aliquots were air-dried in a fume hood and oven-dried in paper bags at $65^{\circ} \mathrm{C}$ under ambient atmosphere for at least $48 \mathrm{~h}$, respectively. In addition, an aliquot of air-dried soil sample (for $2 \mathrm{wk}$ ) was incubated at $25^{\circ} \mathrm{C}$ and remoistened at $75 \%$ of the water holding capacity for $2 \mathrm{wk}$ before analysis.

Soils were analyzed for $\mathrm{pH}$ (1:2.5 in water), redox potential, total $\mathrm{C}, \mathrm{N}$, and $\mathrm{S}$, total organic $\mathrm{C}$ (TOC), and dissolved organic C (DOC). Redox potential was determined with a portable soil redox potentiometer (320P-83, Thermo Orion). Total $\mathrm{C}$ and $\mathrm{N}$ were measured with an elemental analyzer (PE2400, PerkinElmer Inc.). Total organic $\mathrm{C}$ was determined after acid treatment $(10 \% \mathrm{HCl})$ to remove inorganic $\mathrm{C}$, and DOC was extracted with $0.01 \mathrm{~mol} \mathrm{~L}^{-1} \mathrm{NaCl}$ at a soil to solution ratio of 1:20 for $6 \mathrm{~h}$ (Luo et al., 2012). Total $S$ was determined by ICP-AES after digestion with a mixture of $\mathrm{HNO}_{3}$ and $\mathrm{HClO}_{4}(85: 15 \mathrm{v} / \mathrm{v})$ (Zhao and McGrath, 1994). All the above measurements were 
conducted based on freeze-dried samples unless otherwise stated. Selected properties of the soils and the SS used in the work are shown in Table 1.

\section{Chemical Fractionation Analysis}

Inorganic $S$ forms including available and $\mathrm{HCl}$-extractable $\mathrm{S}$ in the soil samples were analyzed using a sequential extraction procedure as follows: $0.016 \mathrm{~mol} \mathrm{~L}^{-1}$ $\mathrm{KH}_{2} \mathrm{PO}_{4}$ (available $\mathrm{S}$ or water-soluble and adsorbed S) (Zhao et al., 2006) and then $1.0 \mathrm{~mol} \mathrm{~L}^{-1} \mathrm{HCl}$ (Hu et al., 2005). All extractions were performed under the same conditions, that is, 1:5 soil/solution ratio $(\mathrm{w} / \mathrm{v})$, shaking for $1 \mathrm{~h}$ at room temperature $\left(25 \pm 2^{\circ} \mathrm{C}\right)$, centrifugation at $7200 \times g$ for $10 \mathrm{~min}$ and filtration through a 0.45 $\mu \mathrm{m}$ cellulose acetate filter. Total and inorganic $\mathrm{S}$ in the $\mathrm{KH}_{2} \mathrm{PO}_{4}$ extracts were determined using ICP-AES and ion chromatography (Dionex DX 500, Dionex Corporation), respectively. Total and inorganic $S$ in the $1.0 \mathrm{~mol} \mathrm{~L}^{-1} \mathrm{HCl}$ extracts was determined with ICP-AES before and after treatment by $S$-free activated charcoal, respectively (Hu et al., 2005). Total organic $S$ was calculated as the difference between total $S$ and inorganic $S$ extracted by $\mathrm{KH}_{2} \mathrm{PO}_{4}$ and $\mathrm{HCl}$ (Zhao and $\left.\mathrm{McGrath}, 1994\right)$.

Organic $S$ fractions including ester sulfate, C-bonded $S$, and residual $S$ were analyzed using a modified Johnson-Nishita distillation-reflux apparatus (Supplemental Fig. S1). More detailed information on the fraction analysis is provided in the supplementary information. In addition, the moisture in the fresh and conditioned soils caused severe reverse flow of $\mathrm{NaOH}$ solution in the Johnson-Nishita apparatus, which limited the analysis of organic $S$ fractions in the samples. Instead, these samples were analyzed after freeze-drying. In this case, the fresh samples were equivalent to the anoxic freeze-dried samples, and the repeated data were not presented in Fig. 1. Each sample was extracted in triplicate.

\section{Sulfur K-edge X-Ray Adsorption Near-Edge Structure Spectroscopy}

The spectral data were collected at Beijing Synchrotron Radiation Facility using the Beamline 4B7A equipped with a $\mathrm{Si}(111)$ monochromator. Spectra were recorded for all samples in the energy range 2450 to $2520 \mathrm{eV}$ in fluorescence mode using a silicon drifted detector (e2v, UK). Energy calibration was performed using a series of reference compounds including $\mathrm{Na}_{2} \mathrm{SO}_{4}$ (at $2482.7 \mathrm{eV}$ ) (Supplemental Fig. S2) and the uncertainty in energy was $0.10 \mathrm{eV}$. The pulverized soil samples (except for the fresh and conditioned samples, which were directly used for the determination after homogenized under $\mathrm{N}_{2}$ atmosphere) were thinly mounted in 2.5- $\mu \mathrm{m}$ Mylar film envelopes and placed into a He purged sample chamber. Depending on the spectrum quality, each XANES spectrum represents an average of three to five scans.

All data were baseline-corrected and normalized to the edge jump, and the contribution of different $S$ species to total $\mathrm{S}$ was deconvoluted over the 2466 to $2489 \mathrm{eV}$ energy range by Gaussian curve fitting (Huffman et al., 1991; Prietzel et al., 2011; Manceau and Nagy, 2012), which was implemented using WinXAS 3.1 (Ressler, 1998). Briefly, six Gaussian functions (G1-G6), each reflecting one functionality, and two arctangent functions, one at low energy for all reduced $S$ forms and another at higher energy for all oxidized forms, were applied for the fitting (Manceau and Nagy, 2012) (Supplemental Table S1). The positions in units of energy $(\mathrm{eV})$ and heights in units of normalized absorbance of the Gaussian and arctangent functions used to fit the spectra were optimized independently. The widths of all the Gaussian and arctangent functions were covaried and then fixed (Manceau and Nagy, 2012). The contribution of each S fraction to total $S$ in the soils was calculated independently from the area under the respective Gaussian peak relative to the total area under the six Gaussian peaks after correcting for the change in absorption cross-section with increasing oxidation state using the generic calibration curve recommended by Manceau and Nagy (2012) (Supplemental Fig. S3). Slight variations in the proportion of $S$ species could be assigned to fits of a given spectra, and an average with coefficient of variation less than $10 \%$ was reported for each $S$ fraction.

\section{Statistics}

Statistical analysis (one-way ANOVA with Tukey pairwise comparison) was conducted using software SPSS 17.0 (SPSS Inc.). Differences between values at $p<0.05$ were considered significantly different.

\section{RESULTS}

\section{General Soil Characteristics}

The soil properties were profoundly affected by the continuous application of SS (Table 1). For example, TOC and DOC contents were increased by $187 \%$ and $143 \%$ in the SS-amended topsoil compared with those in the control topsoil, respectively. Similarly, total S content, ranging from 526 to $1193 \mathrm{mg} \mathrm{kg}^{-1}$, was increased by over $35 \%$ in the SS-amended topsoil compared with that in the control topsoil. Meanwhile, the fluvo-aquic soil, referred to as a calcareous soil, was obviously alkaline, and the $\mathrm{pH}$ tended to be neutral, and the carbonate content was also obviously decreased in the SS-amended soil. Both TOC and total S contents were decreased by 34 to $78 \%$ with soil depth. Apart from output from crop production, parts of the nutrients including $\mathrm{C}, \mathrm{S}$, and $\mathrm{N}$ 

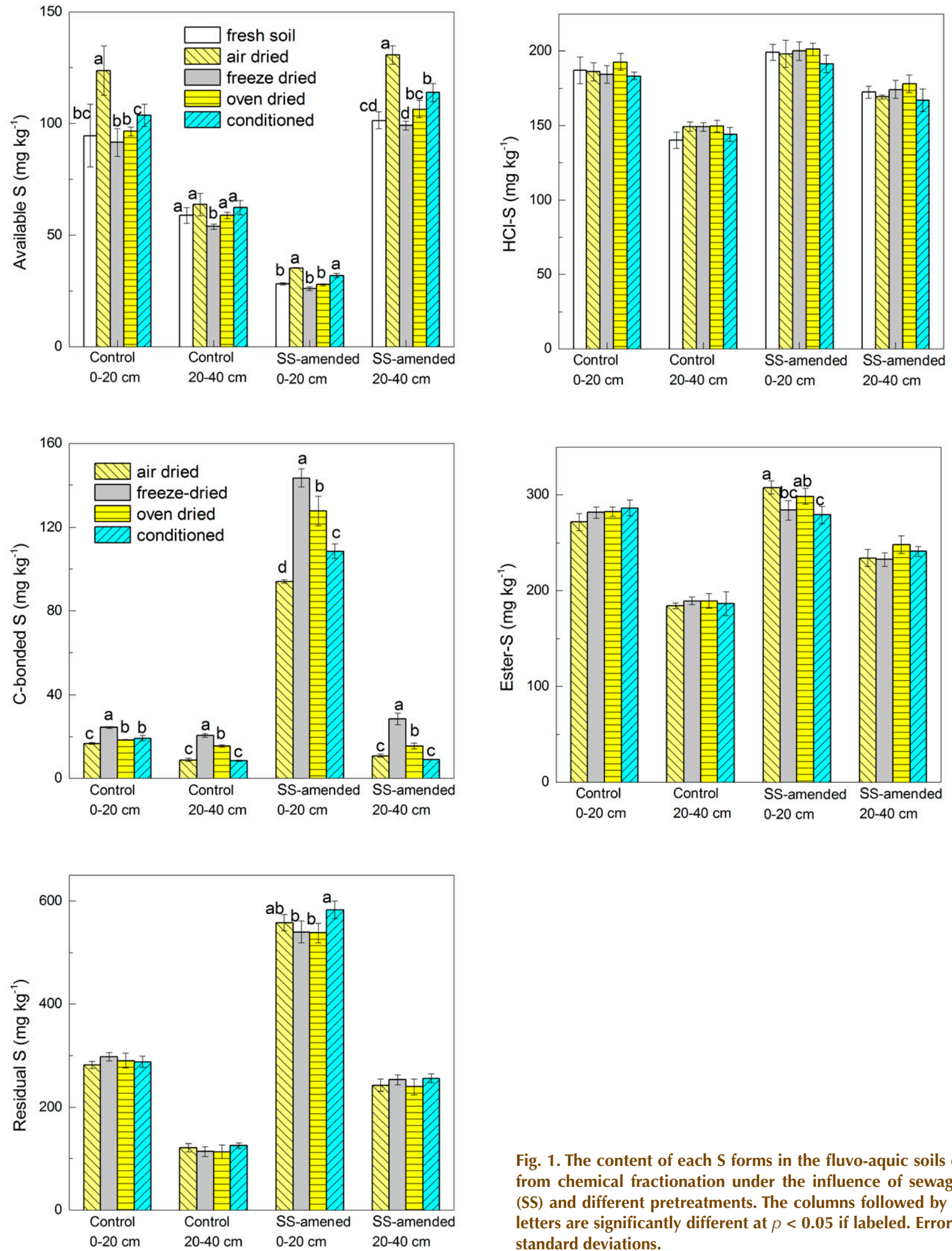

were transported to lower depths through leaching and then accumulated gradually at the subsoil horizon. For example, total $S$ content in the SS-amended subsoil was obviously higher than that in the control subsoil.

Fig. 1. The content of each $S$ forms in the fluvo-aquic soils obtained from chemical fractionation under the influence of sewage sludge (SS) and different pretreatments. The columns followed by different letters are significantly different at $p<0.05$ if labeled. Error bars are standard deviations.

\section{Sulfur Fractionation by Chemical Extraction}

Both the amounts of inorganic and organic $S$ in the soils were markedly affected by the application of SS and soil depth regardless of sample pretreatment (Fig. 1). The available $S$ including water-soluble and adsorbed $S$ in the SS-amended topsoil was significantly lower than that in the control topsoil. In contrast, the available $S$ in the SS-amended subsoil was 
significantly higher than that in the control subsoil. The $\mathrm{HCl}-$ extractable $S$, as the main species of inorganic $S$, accounted for 17.3 to $27.9 \%$ of total $S$ in the soils (based on the freeze-dried samples and the same for the data below), and the proportions of HCl-extractable $S$ to total $S$ in the soils were significantly increased with soil depth. Only slight increases in the contents of the $\mathrm{HCl}$-extractable $S$ in the SS-amended soil were observed, while its proportions to total $S$ were markedly decreased in the SS-amended soil compared with those in the control soil. Organic $S$ was the predominant form of $S$, amounting to over $61 \%$ of total soil S. Among the three organic $S$ forms in the soils, residual $S$ was the main fraction, followed by ester $S$ and then C-bonded S. The application of SS significantly increased the contents of C-bonded S and residual S in the SS-amended topsoil compared with those in the control topsoil when organic $S$ fractions were considered $(p<0.05)$.

Compared with freeze-drying, air-drying significantly increased the content of available $S$ by over $18 \%$ while markedly decreased the content of C-bonded S by over $31 \%$ in the soils (Fig. 1). Conditioning pretreatment also exhibited a similar but less obvious effect on distribution of soil $S$ fractions, particularly on the C-bonded $S$ fraction $(p<0.05)$, to air-drying. Fresh samples showed a similar distribution of inorganic $S$ fractions to freeze-dried samples except for having relatively larger error bars. In contrast, oven-drying showed significant effects on the contents of available $S$ (except for the topsoils) and C-bonded $S$ when compare with freeze-drying $(p<0.05)$. Distributions of the relatively stable fractions (i.e., $\mathrm{HCl}$-extractable $S$ and residual organic $S$ ) were less affected by the pretreatments.

\section{Sulfur Speciation by X-ray Adsorption Near-Edge Structure Spectroscopy}

The $S K$-edge XANES spectra of the soils, presented in Fig. 2, are generally characterized by four distinct signals: one in the energy region around $2474 \mathrm{eV}$, representing reduced organic S; two around 2476 2479 eV, representing organic $S$ in the intermediate oxidation states; and the fourth one around $2482.6 \mathrm{eV}$, representing oxidized organic S (ester S) and inorganic $S$ (sulfate). These consistent features in the XANES spectra allowed us to deconvolute and fit the experimental spectra using a series of Gaussian curves (G1-G6) and the sum of two arctangent functions as shown in Fig. 3 and more detailed information on the Gaussian curves is available in Supplemental Table S1. No reduced inorganic $S$ was detected in the soils. Oxidized $S$ in most cases dominated over the other two fractions except for in the SS-amended topsoil in which reduced organic $S$ was the dominant species (Fig. 3 and 4). It is noteworthy that the signals representing intermediate $S$ significantly increased while those representing reduced organic $S$ tended to decrease with soil depth (Fig. 2). In contrast, the control and SS-amended subsoils showed almost identical distribution of $S$ fractions (Fig. 4).

Similar to the fractionation results, the XANES analysis confirmed that fresh samples exhibited almost identical spectra with freeze-dried samples, while the intensity of the signals
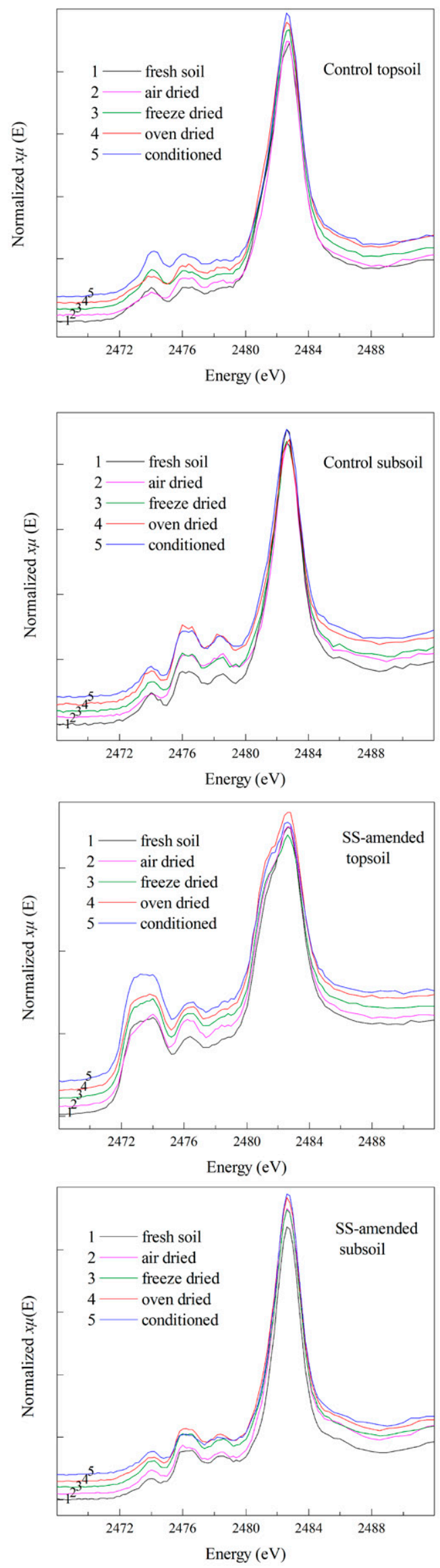

Fig. 2. Stacked S K-edge XANES spectra of the fluvo-aquic soil samples under the influence of sewage sludge (SS) and different pretreatments. 


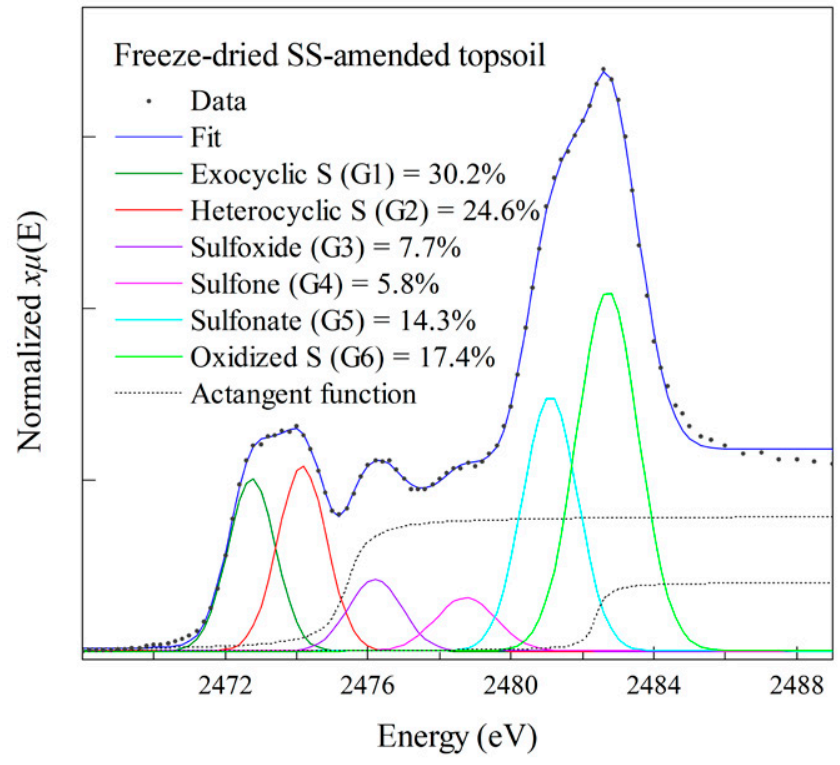

Fig. 3. Spectral deconvolution of the $S K$-edge XANES spectrum from the sewage sludge (SS)-amended topsoil with Gaussian curves (G1 to G6) and two arctangent functions.

representing reduced $S$ compounds in the freeze-dried and conditioned samples tended to increase compared with that in the air-dried samples (Fig. 2). Deconvolution of the spectra of the soil samples showed that air-drying significantly decreased the proportions of reduced organic $S$ by 11 to $24 \%$ and intermediate $S$ fraction by 8 to $27 \%$ while increased the proportion of oxidized $S$ fraction by 12 to $47 \%$ when compared with freeze-drying (Fig. 4). Oven-drying only exerted a weak effect on the distribution of $S$ speciation by decreasing the proportion of reduced organic $S$ by 6 to $16 \%$ in the soils compared with freeze-drying. Conditioning pretreatment tended to increase the proportion of reduced organic $S$ fractions $(p<0.05)$ while decreased the proportion of intermediate $S$ fractions to a certain extent when compared with freeze-drying.

\section{DISCUSSION}

\section{The Impact of Sample Pretreatments}

It is well accepted that freeze-drying pretreatment under anoxic conditions for the redox-sensitive samples (e.g., sediments and anoxic soils) can reduce the unnecessary disturbance by $\mathrm{O}_{2}$ and, thus, provide true information on the distribution of $S$ speciation in such samples. Air-drying could cause the breakdown of complex organic S compounds (mainly C-bonded S) into more labile $\mathrm{S}$ compounds including $\mathrm{SO}_{4}{ }^{2-}$ due to exposure to oxic atmosphere, particularly in the presence of aerobic microbes during the slow drying processes (Wieder et al., 1985; Tan et al., 1994), leading to a significant increase in the contents of DOC and available S (Tan et al., 1994; Jones and Willett, 2006). Under moist conditions ( $75 \%$ of the water holding capacity in the study), the labile $\mathrm{S}$ (mainly C-bonded) was degraded into inorganic $\mathrm{SO}_{4}{ }^{2-}$, while the relatively stable residual $\mathrm{S}$ (also referred to as $\mathrm{C}$-bonded $\mathrm{S}$ by some researchers, e.g., Solomon et al., 2005) tended to increase in the conditioned samples since mineralization and immobilization of $S$ can occur concurrently (Knights et al., 2000). In contrast, microbial activity can be inhibited by fast dehydration of soils when pretreated with oven-drying (Stark and Firestone, 1995). As a result, the labile fraction of $S$, which is highly susceptible to microbial attack (such as C-bonded S and ester S) (Yang et al., 2007; Solomon et al., 2011), was less affected by oven-drying. When fresh samples were first treated with formalin to inhibit microbial activity and then treated with air-drying, similar distribution of $S$ speciation in the soils to those obtained with oven-drying was observed by XANES analysis (data not presented), confirming that parts of the oxidation were biologically mediated (Prietzel et al., 2009). No obvious effect was observed for other $S$ fractions between different pretreatments when chemical fractions were considered, probably due to the relatively stable properties or the chemical alteration caused by fractionation.

The transformation of labile S species (mainly C-bonded S) under different pretreatments based on the chemical fractionation was well consistent with the XANES results. Both reduced and intermediate organic $S$ tended to be transformed into oxidized $\mathrm{S}\left(\mathrm{SO}_{4}{ }^{2-}\right)$ during the slow air-drying process at ambient conditions (Fig. 4). As a result, the proportion of

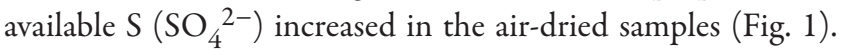
During the conditioning period, parts of intermediate $S$ were deoxidized into reduced $S$, while the proportion of oxidized $S$ was less affected. The decreased Eh in the conditioned SS-amended topsoil sample $(468.6 \mathrm{mV})$ compared with the relevant original field-moist (fresh) sample $(527.2 \mathrm{mV})$ could also facilitate the transformation of intermediate $S$ into reduced forms (Prietzel et al., 2009) and, thus, supported the conclusion. The changes of niche in the conditioned samples by the constantly moist treatment, including the increase of reduced $S$ content, the decrease of redox potential, and microbial activity, probably make it fail to reflect the true distribution of $S$ speciation in soils and might be one of the reasons for the differences between field and pot experiments (Pamidi et al., 2001; McLaughlin et al., 2006). Concomitant with the significant decrease in C-bonded $S$, the proportions of reduced $S$ were also slightly decreased (by 6-16\%) in the oven-dried samples. Prietzel et al. (2009) indicated that oven-drying pretreatment caused significant changes of $S$ speciation in the soils, which contained considerable amounts of reduced inorganic $S$. In the present work, no reduced inorganic $S$ was detected in the soils and organic $S$ was believed to be relatively stable at the ambient conditions (Novák and Wieder, 1992; Hutchison et al., 2001). As a result, less impact was observed for the oven-dried samples when compared with the freezedried samples. In contrast, Hinckley et al. (2011) claimed that there was no difference in soil $S$ speciation between oven- and freeze-drying treatments. Such difference between Hinckley's and our results might be related to the anoxic atmosphere during the freeze-drying pretreatment and the following analysis adopted in the present work, which ruled out any possible biotic changes of $S$ speciation that oxic pretreated soils usually suffered from (Wieder et al., 1996). It is expectable that if oven-dried 
samples were conducted under anoxic conditions for the entire pretreatment, $S$ speciation could be also preserved as did in the anoxic freeze-dried samples.

Based on the changes of $S$ fractions obtained by fractionation and of $S$ oxidation states observed by XANES analysis, it can be assumed that oxic air-drying, a popular pretreatment method for soils, might fail to reflect the true distribution of $S$ speciation by underestimating the contribution of reduced $S$ in soils and, thus, lead to a wrong assumption concerning the role of $S$ in the process of redox and the immobilization of heavy metals (relating to reduced thiol) in the environment. In addition, although fresh field-moist samples showed almost identical distribution of $S$ speciation to the freeze-dried samples when conducted under anoxic conditions, the results were highly dependent on the water content in the soils and would incur large errors, particularly for chemical fractionation analysis. Fresh samples are, thus, unsuitable for direct analysis of $S$ speciation. The entire pretreatment process from sampling to drying, grinding, and analysis under anoxic atmospheres is, therefore, required for accurate $S$ speciation analysis. Compared with oven-drying under anoxic conditions, the anoxic freeze-drying is operationally easier and, thus, recommended. The following discussion is based on the results obtained from the anoxic freeze-dried samples.

\section{Effects of Sewage Sludge Application}

Due to continuous application of S-containing fertilizers (i.e., $\mathrm{Ca}$ superphosphate, which contains about $10 \% \mathrm{~S}$ as $\mathrm{CaSO}_{4}$ ) and $\mathrm{S}$-rich SS, the total $\mathrm{S}$ was significantly increased in the soils. Meanwhile, the content of available $S$ in the control topsoil amounted to $91.6 \mathrm{mg} \mathrm{kg}^{-1}$, far higher than the previous published data for the Chinese agricultural soils ( $\mathrm{Hu}$ et al., 2005) and equivalent to the levels in the long-term fertilized soils with S-containing fertilizers (Xu et al., 2014). Contrasting with previous research (Yang et al., 2007; Förster et al., 2012), the available $S$ was not increased in the SS-amended topsoil and only amounted to $26.1 \mathrm{mg} \mathrm{kg}^{-1}$. The relatively lower content of available $S$ could be ascribed to the following two reasons. First, the application of SS brought large numbers of organic ligands and significantly increased the content of DOC in the topsoil (Table 1) and, thus, facilitated the transportation of $S$ into lower depths since the inorganic sulfate $\left(\mathrm{SO}_{4}{ }^{2-}\right)$ is more prone to leaching and a competition of adsorption sites occurred between the electronegative ligands and $\mathrm{SO}_{4}{ }^{2-}$ (Guggenberger and Zech, 1992). With the decrease of DOC in the subsoil, the available $S$ were gradually sequestrated in the horizon and amounted to nearly $100 \mathrm{mg} \mathrm{kg}^{-1}$ (Fig. 1). Second, the higher crop yield in the SS-amended soil exhausted more available $S$ in the topsoil compared with the control soil since the crop yield in the SS-amended block was nearly tripled compared with that in the control block $\left(16,051 \mathrm{~kg} \mathrm{ha}^{-1}\right.$ vs. $5955 \mathrm{~kg} \mathrm{ha}^{-1}$, based on the yields of wheat plus maize in 2011, not published). As a calcareous soil, parts of inorganic $\mathrm{S}$ (i.e., $\mathrm{HCl}$-extractable $\mathrm{S}$ ) were accumulated as a coprecipitate with $\mathrm{CaCO}_{3}$ (Hu et al., 2005 ) and became the predominant fraction of inorganic $S$ in
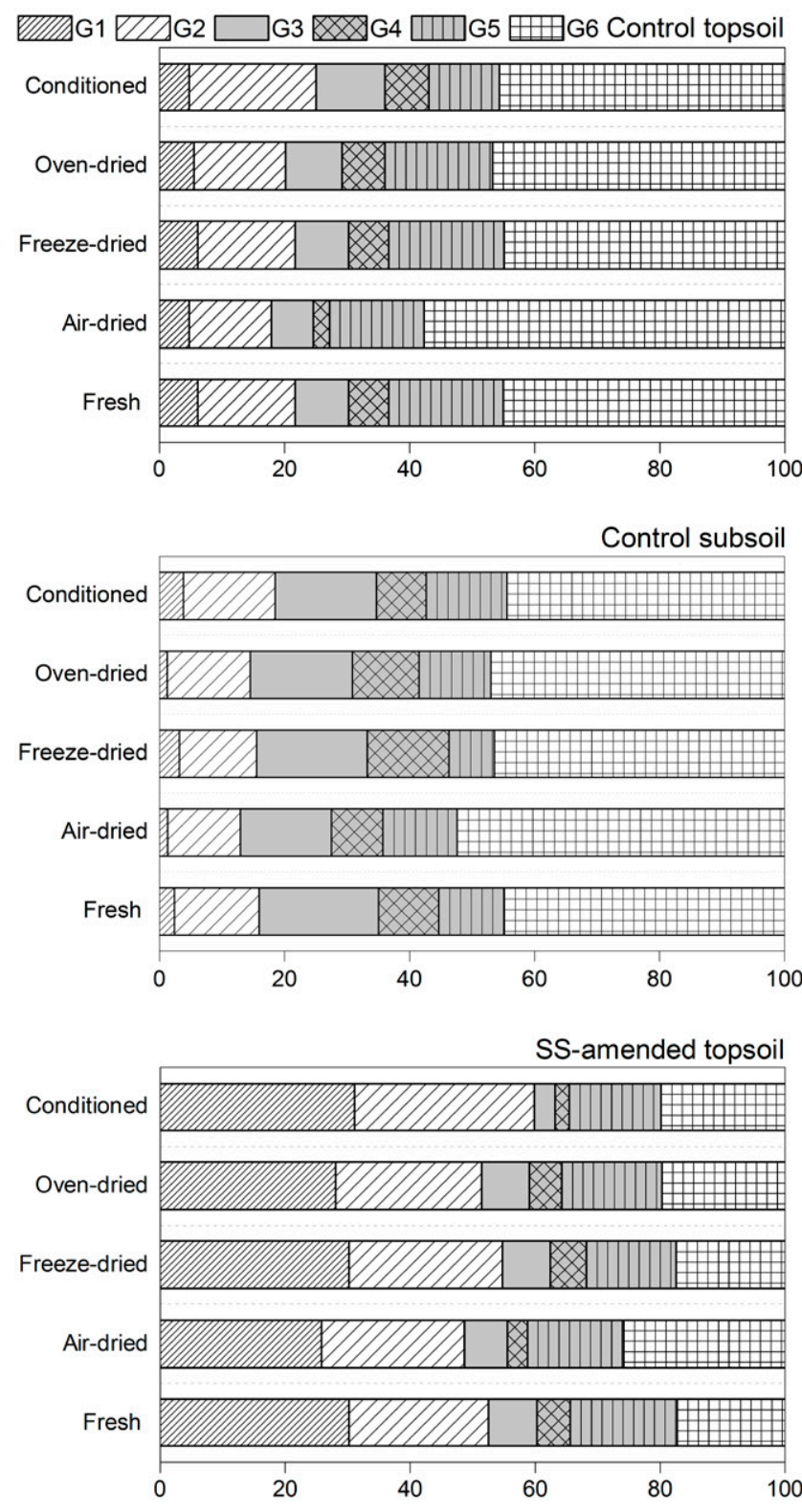

SS-amended subsoil

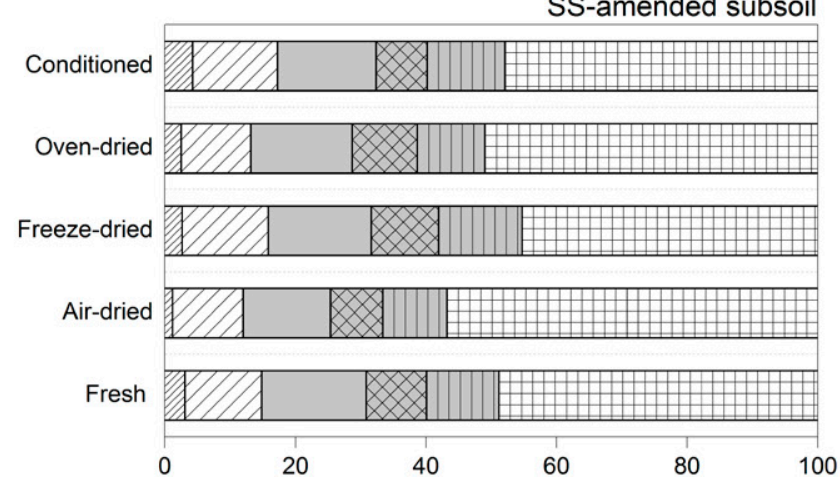

Fig. 4. Percentage (\%) of reduced (G1 and G2), intermediate (G3, G4, and G5), and oxidized $\mathrm{S}$ forms in the fluvo-aquic soils affected by sewage sludge (SS) amendment and different pretreatments, as calculated by spectral deconvolution by Gaussian curve fitting and subsequently corrected for absorption cross-section from the $S K$-edge XANES spectra.

the soil. The organic ligands present in the SS could dissolute and complex $\mathrm{Ca}^{2+}$ in the calcareous soil, evidenced by the decrease of carbonate content in the SS-amended soil (Table 1), and thus 
inhibited the accumulation of $\mathrm{HCl}$-extractable $\mathrm{S}$. As a result, the content of $\mathrm{HCl}$-extractable $S$ was only slightly increased although the total soil $S$ content was significantly increased in the SS-amended soil (Fig. 1).

Consistent with previous reports (Yang et al., 2007; Förster et al., 2012), the continuous application of SS also facilitated the accumulation of $\mathrm{C}$-bonded $\mathrm{S}$ and residual $\mathrm{S}$ in the soils compared with the control soil amended with only $\mathrm{P}$ and $\mathrm{K}$ fertilizers, which was also evidenced by the significant increase in the proportions of reduced and intermediate organic $S$ in the topsoil (Fig. 4). The obvious accumulation of C-bonded and residual $S$ in the SS-amended soil could be ascribed mainly to the high organic $\mathrm{C}$ content in the SS and secondarily to the direct input of the organic $S$ from the applied SS. It was confirmed that the proportions of reduced and intermediate organic $S$ in the SS were significantly lower than those in the SS-amended soil (Supplemental Fig. S4) although a large amount of S was introduced into the soil through the application of SS, indicating the higher proportions of the reduced and intermediate organic $S$ in the soil should be ascribed to the biochemical processes occurred in the soil. It is proposed that the application of carbonrich materials increased the microbial activity and, thus, facilitated the accumulation of the organic $S$, particularly C-bonded $S$ and residual $S$ in soils (Ghani et al., 1992; Eriksen, 1996; Knights et al., 2000). Meanwhile, although previous work suggested that the input of inorganic $S$ facilitated the accumulation of ester $S$ in soils (Solomon et al., 2011), the lower levels of inorganic $S$ were preserved due to crop uptake and leaching effect and, thus, contributed little to the incorporation of $\mathrm{S}$ into soil organic C, that is, ester S, in the SS-amended soil (Maynard et al., 1985). In addition, due to the low level of TOC in the SS-amended subsoil, the microbial activity was, consequently, inhibited, and thus the accumulation of organic $S$ was limited (Fig. 1 and 4) although total $S$ and available $S$ were significantly increased in the horizon. As a result, the SS-amended subsoil showed almost similar $S$ fraction distribution to the control subsoil, indicating that the accumulation of organic S, particularly of $\mathrm{C}$-bonded $\mathrm{S}$, was positively related to the content of TOC in soils (Hu et al., 2005; Xu et al., 2014).

\section{CONCLUSIONS}

The present study demonstrated with solid evidence that different sample pretreatments artificially caused significant changes on $S$ speciation in the well-aerated soil in which the species of $S$ were believed to be relatively stable, and proposed that an anoxic atmosphere for the pretreatment from sampling to analysis for the redox-sensitive samples is necessary and the anoxic freeze-drying pretreatment is particularly recommended. Furthermore, the study provided detailed information on the influence of SS application on S speciation in the calcareous soil. Continuous application of SS (for $7 \mathrm{yr}$ since 2006) resulted in a significant decrease of available $S$ and facilitated the transportation of the available $S$ along the soil profile. Another distinct change in $S$ speciation with the application of SS was the significant accumulation of organic S, particularly C-bonded $S$ and residual S, suggesting parts of inorganic $S$ and organic ester $S$ from inorganic fertilizer and SS were transformed into the C-bonded $S$ in the soils through abiotic and biotic processes, and the mechanisms involved in the transformation deserve further research.

\section{SUPPLEMENTARY INFORMATION}

Supplementary information associated with this article includes: $S$ species related to Gaussian curves, details of organic $S$ fraction analysis, scaling factors for correction of $S$ fractions from Gaussian curve fitting, and XANES spectrum of sewage sludge applied in the study.

\section{ACKNOWLEDGMENTS}

The work was funded by 973 Program of China (Grant No. 2014CB 441102), the Strategic Priority Research Program of the Chinese Academy of Sciences (XDB14020202), and the National Natural Science Foundation of China (Grant No. 41023005 and 21277157). We are grateful to Dr. Yidong Zhao at the beamline 4B7A of Beijing Synchrotron Radiation Facility for providing excellent support.

\section{REFERENCES}

Eriksen, J. 1996. Incorporation of S into soil organic matter in the field as determined by the natural abundance of stable $S$ isotopes. Biol. Fertil. Soils 22:149-155 10.1007/BF00384447. doi:10.1007/BF00384447

Förster, S., G. Welp, and H.W. Scherer. 2012. Sulfur specification in bulk soil as influenced by long-term application of mineral and organic fertilizers. Plant Soil Environ. 58:316-321.

Ghani, A., R.G. McLaren, and R.S. Swift. 1992. Sulphur mineralisation and transformations in soils as influenced by additions of carbon, nitrogen and sulphur. Soil Biol. Biochem. 24:331-341. doi:10.1016/0038$\underline{0717(92) 90193-2}$

Guggenberger, G., and W. Zech. 1992. Retention of dissolved organic carbon and sulfate in aggregated acid forest soils. J. Environ. Qual. 21:643-653. doi: $10.2134 /$ jeq1992.00472425002100040019x

Hashimoto, Y., and N. Yamaguchi. 2013. Chemical speciation of cadmium and sulfur K-edge XANES spectroscopy in flooded paddy soils amended with zerovalent iron. Soil Sci. Soc. Am. J. 77:1189-1198. doi:10.2136/ sssaj2013.01.0038

Hinckley, E.-L.S., S. Fendorf, and P. Matson. 2011. Short-term fates of high sulfur inputs in Northern California vineyard soils. Nutr. Cycling Agroecosyst. 89:135-142. doi: 10.1007/s10705-010-9383-3

$\mathrm{Hu}$, Z.Y., F.J. Zhao, and S.P. McGrath. 2005. Sulphur fractionation in calcareous soils and bioavailability to plants. Plant Soil 268:103-109. doi:10.1007/ s11104-004-0229-0

Huffman, G.P., S. Mitra, F.E. Huggins, N. Shah, S. Vaidya, and F. Lu. 1991. Quantitative analysis of all major forms of sulfur in coal by X-ray absorption fine structure spectroscopy. Energy Fuels 5:574-581. doi:10.1021/ ef00028a008

Hutchison, K.J., D. Hesterberg, and J.W. Chou. 2001. Stability of reduced organic sulfur in humic acid as affected by aeration and $\mathrm{pH}$. Soil Sci. Soc. Am. J. 65:704-709. doi:10.2136/sssaj2001.653704x

Jones, D.L., and V.B. Willett. 2006. Experimental evaluation of methods to quantify dissolved organic nitrogen (DON) and dissolved organic carbon (DOC) in soil. Soil Biol. Biochem. 38:991-999. doi:10.1016/j. soilbio.2005.08.012

Kirchmann, H., F. Pichlmayer, and M.H. Gerzabek. 1996. Sulfur balances and sulfur-34 abundance in a long-term fertilizer experiment. Soil Sci. Soc. Am. J. 60:174-178. doi:10.2136/sssaj1996.03615995006000010028x

Knights, J.S., F.J. Zhao, B. Spiro, and S.P. McGrath. 2000. Long-term effects of land use and fertilizer treatments on sulfur cycling. J. Environ. Qual. 29:1867-1874. doi:10.2134/jeq2000.00472425002900060020x

Li, Q., X.Y.Guo, X.H.Xu, Y.B.Zuo, D.P. Wei, and Y.B.Ma.2012.Phytoavailability of copper, zinc and cadmium in sewage sludge-amended calcareous soils. Pedosphere 22:254-262. doi:10.1016/S1002-0160(12)60012-5 
Luo, L., S. Lin, H.L. Huang, and S.Z. Zhang. 2012. Relationships between aging of PAHs and soil properties. Environ. Pollut. 170:177-182. doi:10.1016/j. envpol.2012.07.003

Manceau, A., and K.L. Nagy. 2012. Quantitative analysis of sulfur functional groups in natural organic matter by XANES spectroscopy. Geochim. Cosmochim. Acta 99:206-223. doi:10.1016/j.gca.2012.09.033

Maynard, D.G., J.W.B. Stewart, and J.R. Bettany. 1985. The effects of plants on soil sulfur transformations. Soil Biol. Biochem. 17:127-134. doi:10.1016/0038-0717(85)90103-8

McLaughlin, M.J., M. Whatmuff, M. Warne, D. Heemsbergen, G. Barry, M. Bell, D. Nash, and D. Pritchard. 2006. A field investigation of solubility and food chain accumulation of biosolid-cadmium across diverse soil types. Environ. Chem. 3:428-432 10.1071/EN06061. doi:10.1071/EN06061

Novák, N., and R.K. Wieder. 1992. Inorganic and organic sulfur profiles in nine sphagnum peat bogs in the United States and Czechoslovakia. Water Air Soil Pollut. 65:353-369. doi:10.1007/BF00479898

Pamidi, J., K.M. Goh, and R.G. McLaren. 2001. Comparison of three different methods of determining soil sulphur mineralization in relation to plant sulphur availability in soils. Biol. Fertil. Soils 34:131-139. doi:10.1007/ $\underline{5003740100378}$

Prietzel, J., A. Botzaki, N. Tyufekchieva, M. Brettholle, and J. Thieme. 2011. Sulfur speciation in soil by $S$ K-edge XANES spectroscopy: Comparison of spectral deconvolution and linear combination fitting. Environ. Sci. Technol. 45:2878-2886. doi:10.1021/es102180a

Prietzel, J., J. Thieme, M. Salomé, and H. Knicker. 2007. Sulfur K-edge XANES spectroscopy reveals differences in sulfur speciation of bulk soils, humic acid, fulvic acid, and particle size separates. Soil Biol. Biochem. 39:877890. doi:10.1016/j.soilbio.2006.10.007

Prietzel, J., N. Tyufekchieva, K. Eusterhues, I. Kögel-Knabner, J. Thieme, D. Paterson, I. McNulty, M. De Jonge, D. Eichert, and M. Salomé. 2009. Anoxic versus oxic sample pre-treatment: Effects on the speciation of sulfur and iron in well aerated and wetland soils as assessed by $\mathrm{X}$-ray absorption near-edge spectroscopy (XANES). Geoderma 153:318-330. doi:10.1016/j.geoderma.2009.08.015

Ressler, T. 1998. WinXAS: A program for X-ray absorption spectroscopy data analysis under MS-Windows. J. Synchrotron Radiat. 5:118-122. doi:10.1107/S0909049597019298

Solomon, D., J. Lehmann, K.K. de Zarruk, J. Dathe, J. Kinyangi, B. Liang, and
S. Machado. 2011. Speciation and long- and short-term molecular-level dynamics of soil organic sulfur studied by $\mathrm{X}$-ray absorption near-edge structure spectroscopy. J. Environ. Qual. 40:704-718. doi:10.2134/ jeq2010.0061

Solomon, D., J. Lehmann, I. Lobe, C.E. Martínez, S. Tveitnes, C.C. Du Preez, and W. Amelung. 2005. Sulphur speciation and biogeochemical cycling in long-term arable cropping of subtropical soils: Evidence from wet-chemical reduction and S K-edge XANES spectroscopy. Eur. J. Soil Sci. 56:621-634. doi:10.1111/j.1365-2389.2005.00702.x

Stark, J.M., and M.K. Firestone. 1995. Mechanisms for soil moisture effects on activity of nitrifying bacteria. Appl. Environ. Microbiol. 61:218-221.

Tan, Z., R.G. McLaren, and K.C. Cameron. 1994. Forms of sulfur extracted from soils after different methods of sample preparation. Aust. J. Soil Res. 32:823-834. doi:10.1071/SR9940823

Wieder, R.K., G.E. Lang, and V.A. Granus. 1985. An evaluation of wet chemical methods for quantifying sulfur fractions in freshwater wetland peat. Limnol. Oceanogr. 30:1109-1115. doi:10.4319/lo.1985.30.5.1109

Wieder, R.K., M. Novák, and D. Rodrigues. 1996. Sample drying, total sulfur, and stable sulfur isotopic ratio determination in freshwater wetland peat. Soil Sci. Soc. Am. J. 60:949-952. doi:10.2136/ sssaj1996.03615995006000030038x

Xia, K., F. Weesner, W.F. Bleam, P.R. Bloom, U.L. Skyllberg, and P.A. Helmke. 1998. XANES studies of oxidation states of sulfur in aquatic and soil humic substances. Soil Sci. Soc. Am. J. 62:1240-1246. doi:10.2136/ sssaj1998.03615995006200050014x

Xu, C., S.S. Wang, J.M. Li, Y.B. Ma, W.T. Sun, L. Luo, and S.Z. Zhang. 2014. Effect of long-term fertilization on evolution of $S$ forms in a red soil and a black soil. (in Chinese.) Chinese J. Appl. Ecol. 25:1069-1075.

Yang, Z.H., B.R. Singh, S. Hansen, Z.Y. Hu, and H. Riley. 2007. Aggregate associated sulfur fractions in long-term ( $>80$ years) fertilized soils. Soil Sci. Soc. Am. J. 71:163-170. doi:10.2136/sssaj2006.0242

Zhao, F.J., J. Lehmann, D. Solomon, M.A. Fox, and S.P. McGrath. 2006. Sulphur speciation and turnover in soils: Evidence from sulphur K-edge XANES spectroscopy and isotope dilution studies. Soil Biol. Biochem. 38:10001007. doi:10.1016/j.soilbio.2005.08.013

Zhao, F.J., and S.P. McGrath. 1994. Extractable sulphate and organic sulphur in soils and their availability to plants. Plant Soil 164:243-250. doi:10.1007/ $\underline{\mathrm{BF} 00010076}$ 